\title{
Adding systematic biopsy to magnetic resonance ultrasound fusion targeted biopsy of the prostate in men with previous negative biopsy or enrolled in active surveillance programs
}

\section{A prospective single center, randomized study}

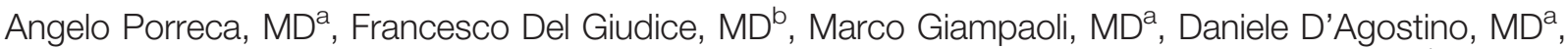 \\ Daniele Romagnoli, MD ${ }^{a}$, Paolo Corsi, $\mathrm{MD}^{\mathrm{a}}$, Alessandro Del Rosso, $\mathrm{MD}^{\mathrm{a}}$, Martina Maggi, $\mathrm{MD}^{\mathrm{b}}$, \\ Benjamin I. Chung, $\mathrm{PhD}^{\mathrm{c}}$, Matteo Ferro, MD, PhD ${ }^{\mathrm{d}}$, Ottavio de Cobelli, MD ${ }^{\mathrm{d}, \mathrm{e}}$, Giuseppe Lucarelli, MD, PhD (i), \\ Riccardo Schiavina, $\mathrm{MD}^{\mathrm{g}}$, Ettore De Berardinis, $\mathrm{MD}^{\mathrm{b}}$, Alessandro Sciarra, MD ${ }^{\mathrm{b}}$, Gian Maria Busetto, MD, PhD ${ }^{\mathrm{b}, *}$
}

\begin{abstract}
Magnetic resonance imaging (MRI) targeted biopsy (TBx) of the prostate demonstrated to improve detection rate (DR) of clinically significant prostate cancer (csPCa) in biopsy-naive patients achieving strong level of evidence. Nevertheless, the csPCa yield for TBx alone versus TBx plus systematic biopsy (SBx) after accounting for overlapping of SBx cores with TBx cores, in prior-negative or active surveillance (AS) patients has not been well established.

The objective of the study was to investigate benefits in terms of detection rate and pathological stratification of prostate cancer (PCa) using contextual SBx during MRI-TBx.

Patients previously submitted to negative-SBx (cohort A) and those enrolled in an AS program (cohort B) who showed at least 1 suspicious area with a PIRADSv2 score $\geq 3$ were prospectively and randomly assigned to only TBx strategy versus TBx plus SBx strategy. SBx locations could not encompass the TBx sites, so that the results of each type of biopsy were independent and did not overlap.

A total of 312 patients were included in the 2 cohorts (cohort A: 213 cases; cohort B: 99 cases). No significant differences were found in terms of overall PCa-DR (77.6\% vs 69.6\% respectively; $P=.36)$ and csPCa-DR (48.2\% vs 60.9 respectively; $P=.12)$. The $\mathrm{MRI}-\mathrm{TBx}$ alone cohort showed higher csPCa/PCa ratio (87.5\% vs $62.2 \% ; P=.03)$. The MRI-TBx plus SBx group subanalysis showed significantly higher csPCa-DR obtained at the MRI-TBx cores when compared with the SBx cores (43.7\% vs $24.1 \%$, respectively; $P=.01)$. Independently to age, prostatic-specific antigen and prostate imaging-reporting and data system score, either in rebiopsy (OR 0.43, 0.21-0.97) or AS (OR 0.46, 0.32-0.89) setting, SBx cores were negatively associated with the csPCa-DR when combined to TBx cores.

MRI-TBx should be considered the elective method to perform prostate biopsy in patients with previous negative SBx and those considered for an AS program. Adding SBx samples to MRI-TBx did not improve detection rate of csPCa.

Abbreviations: $A S=$ active surveillance, $\operatorname{csPCa}=$ clinically significant prostate cancer, $\mathrm{DR}=$ detection rate, $\mathrm{FBx}=$ fusion biopsy, $\mathrm{MRI}=$ magnetic resonance imaging, $\mathrm{PCa}=$ prostate cancer, $\mathrm{SBx}=$ systematic biopsy, $\mathrm{TBx}=$ targeted biopsy, TSE $=$ turbo spin echo.
\end{abstract}

Keywords: biopsy, clinically significant, detection rate, magnetic resonance imaging, prostate biopsy, prostate cancer

\footnotetext{
Editor: Giandomenico Roviello.

The authors have no conflicts of interest to disclose.

The datasets generated during and/or analyzed during the current study are available from the corresponding author on reasonable request.

a Department of Urology, Policlinico Abano Terme, Abano Terme (PD), ' Department of Maternal-Child and Urological Sciences, "Sapienza" Rome University, Policlinico Umberto I Hospital, Rome, ${ }^{c}$ Department of Urology, European Institute of Oncology (IEO), Milan, Italy, ${ }^{d}$ Department of Urology, European Institute of Oncology, IRCCS, ${ }^{e}$ Department of Oncology and Hemato-Oncology, University of Milan, Milan, ${ }^{f}$ Department of Emergency and Organ Transplantation-Urology, Andrology and Kidney Transplantation Unit, University of Bari, Bari, ${ }^{9}$ Department of Urology, Alma Mater Studiorum Bologna, Policlinico S. Orsola Malpighi, Bologna, Italy.

*Correspondence: Gian Maria Busetto, Sapienza University of Rome, Policlinico Umberto I, Viale del Policlinico, 155, 00185 Rome, Italy

(e-mail: gianmaria.busetto@uniroma1.it)

Copyright $\odot 2020$ the Author(s). Published by Wolters Kluwer Health, Inc.

This is an open access article distributed under the terms of the Creative Commons Attribution-Non Commercial License 4.0 (CCBY-NC), where it is permissible to download, share, remix, transform, and buildup the work provided it is properly cited. The work cannot be used commercially without permission from the journal. How to cite this article: Porreca A, Del Giudice F, Giampaoli M, D’Agostino D, Romagnoli D, Corsi P, Del Rosso A, Maggi M, Chung BI, Ferro M, de Cobelli O, Lucarelli G, Schiavina R, De Berardinis E, Sciarra A, Busetto GM. Adding systematic biopsy to magnetic resonance ultrasound fusion targeted biopsy of the prostate in men with previous negative biopsy or enrolled in active surveillance programs: A prospective single center, randomized study. Medicine 2020;99:37(e22059).

Received: 24 February 2020 / Received in final form: 14 July 2020 / Accepted: 5 August 2020

http://dx.doi.org/10.1097/MD.0000000000022059
} 


\section{Introduction}

Prostate cancer $(\mathrm{PCa})$ is the most common neoplasm diagnosed in men. ${ }^{[1,2]}$ Magnetic resonance imaging (MRI) has shown a remarkable accuracy in the detection of clinical significant prostate cancer (csPCa). ${ }^{[3-5]}$ A growing body of evidence suggests that multiparametric $(\mathrm{mp})$ magnetic resonance imaging can improve prostate cancer risk group classification and could reduce false-negative rates and the necessity of repeat biopsies in both biopsy-naive patients and those with prior negativebiopsy; ${ }^{[6-9]}$ not surprisingly, MRI targeted biopsies (TBx) should be strongly considered for any patient, biopsy naive or with a prior negative biopsy who has persistent clinical suspicion of PCa. Techniques for TBx include visual estimation TRUS-GB (cognitive technique), software coregistered MRI-ultrasound fusion (fusion technique), and in-bore MRI-guided biopsy. ${ }^{[10]}$

The use of MR-ultrasound fusion biopsy (FBx) in men with elevated serum prostate-specific antigen (PSA) is becoming increasingly widespread in clinical practice. ${ }^{[1]}$ Prostatic MRI allows the identification of suspicious regions that may be missed by systematic biopsies $(\mathrm{SBx})$ and direct sampling via FBx. ${ }^{[12]}$ As stated by European Association of Urology (EAU) guidelines, MRI-TBx can be used in 2 different diagnostic pathways: the combined pathway in which patients with a positive mpMRI undergo combined $\mathrm{SBx}$ and $\mathrm{TBx}$ and patients with negative mpMRI undergo systematic biopsy; the MR pathway in which patients with a positive mpMRI undergo only TBx and patients with negative multiparametric MRI are not biopsied. ${ }^{[13]}$

Adding MRI TBx to SBx in biopsy naive patients increases the number of ISUP $\geq 2 \mathrm{PCa}$ by approximately $20 \%$ whereas in the repeat-biopsy setting by approximately $40 \%$. Therefore, it has been shown that $\mathrm{TBx}$ improves the detection of clinically significant prostate cancer. ${ }^{[14,15]}$

However, the csPCa yield for TBx alone versus TBx plus SBx after accounting for overlapping of SBx cores with TBx cores has not been well studied.

The aim of our study was to investigate the potential benefit in terms of Detection Rate and pathological stratification of prostate cancer using a contextual SBx during an MRI-TRUS TBx in a 2-cohort population: patients with previous negative SBx and patients considered for an active surveillance (AS) program. ${ }^{[16]}$

\section{Methods}

\subsection{Study population}

This is a prospective randomized single center study approved by our Internal Review Board of Policlinico Abano Terme, Abano Terme (PD), Italy, in accordance with good clinical practice guidelines and ethical principles of the Declaration of Helsinki. An informed consent was obtained from all patients enrolled in the study.

Two different cohorts were considered with the following inclusion criteria: a raised PSA serum level with a previous negative SBx; an enrollment in an AS program for low-risk PCa. In both 2 cohorts, all patients were submitted to mpMRI with at least 1 suspicious area with a PIRADSv2 score $\geq 3$. Between April 2017 and July 2019, 213 consecutive patients were included in the cohort A and 99 consecutive patients in the cohort B.

Cohort A: all patients were previously submitted to SBx for clinical suspicion of prostate cancer based on raised PSA serum level, the histological examination resulted negative for PC and
PSA levels continued to rise. All cases underwent mpMRI and showed at least 1 suspicious area with a PIRADS v2 score $\geq 3$.

Cohort B: all patients were enrolled in an active surveillance program for diagnosis of low-risk (Gleason Score 3+3) PCa within the past year. The diagnosis was obtained by a standard ultrasound guided biopsy and all cases went mpMRI before confirmatory biopsy and showed at least 1 suspicious area with a PIRADS v2 score $\geq 3$.

\subsection{Multiparametric magnetic resonance imaging analysis}

All multiparametric MRI examinations were performed with a 1.5 T whole body scanner (Achieva XR; Philips Medical Systems, Best, the Netherlands) with a 32-channels phased-array surface coil with endorectal coil. After local 3-plane acquisition, required for the correct positioning of the sequences, the morphological and functional studies were carried out. Morphological study of the prostate gland was obtained with Turbo Spin Echo (TSE) T2weighted sequences (TE $100 \mathrm{msec}$, TR $4074 \mathrm{msec}$, slice thickness $3 \mathrm{~mm}$, slice spacing $0.3 \mathrm{~mm}$, field of view [FOV] $180 \times 180 \mathrm{~mm}$ and matrix size $276 \times 205$ ) in the sagittal, axial, and coronal planes, including seminal vesicles and the entire prostate gland. For the functional study, DWI, DCE-MRI, and MRS acquisition were performed. The DWI acquisition was carried out in the axial plane, using a single-shot echo-planar imaging sequence, with 3 b-values $\left(0,600\right.$, and $\left.1500 \mathrm{~s} / \mathrm{mm}^{2}\right)$, slice thickness of $3 \mathrm{~mm}$, FOV $180 \times 180 \mathrm{~mm}$ and matrix size $80 \times 71$. The DCE-MRI was obtained using three-dimensional (3D) T1W high-resolution isotropic volume examination sequence during the intravenous injection of a contrast bolus of $0.1 \mathrm{mmol}$ per kilogram of body weight of Meglumine gadobenate (Multihance, Bracco Diagnostics, Milan, Italy), at flow rate of $3.5 \mathrm{~mL} / \mathrm{s}$ followed by $15 \mathrm{~mL}$ of saline solution. Twenty-three 3D data sets, 1 before and 22 after contrast administration, were acquired with 10 seconds temporal resolution and a total duration of 4 minutes (depending on the volume of the prostate gland). The first data set acquired before contrast agent administration can be used to detect residual blood of previous biopsy. The MRS was obtained with the use of $3 \mathrm{D}$ chemical shift imaging sequence and the following parameters: matrix $10 \times 10 \times 12$ phase-encoding steps with nominal voxel size $<0.5 \mathrm{cc}$; spectral selective suppression of water and lipid signals; interactive automatic shimming up to a line width at half height of the water resonance peak between 15 and $20 \mathrm{~Hz}$. The volume of interest is aligned to axial T2WIs and centered on each prostate to maximize coverage of the whole gland, while minimizing contamination by surrounding tissue. Finally, a TSE T2-weighted sequence (TE $100 \mathrm{msec}$, TR 3445 msec, slice thickness $4 \mathrm{~mm}$, slice spacing $0.4 \mathrm{~mm}$, FOV $260 \times 260$ $\mathrm{mm}$ and matrix size $260 \times 178)$ in the axial plane was acquired from the aortic bifurcation to the symphysis pubis to evaluate the pelvic lymph nodes and bone. All the multiparametric-MRI images were assessed by 1 reader (M.V.) with 10 years of specific experience on prostate MRI who was blinded to all patient information. The DWI and DCE-MRI images were processed on an independent workstation with dedicated software (View Forum, Philips Medical Systems, Best, the Netherlands). Regions of interest positioned on the suspected areas were used to calculate the corresponding value of the apparent diffusion coefficient for DWI. Semiquantitative MRI perfusion was performed on the same workstation with analysis of DCE datasets and signal intensity-time (I-T) curves generation. All lesions were scored using the PI-RADS-v2 according to the ESUR 
guidelines for the evaluation and reporting of prostate multiparametric-MRI. ${ }^{[17,18]}$

\subsection{Conduct of the biopsy}

The biopsies were performed within 3 weeks from the diagnostic mpMRI study by a single urologist with a 5 years' experience in TRUS-guided SBx and TBx. In the cohort B, biopsies were performed at 1 year from inclusion in the AS program as confirmatory biopsies. All patients underwent an MRI-TRUS TBx on suspicious target lesions at mpMRI (PIRADSv2 score 35) using the Artemis platform. After TBx, a SBx was performed or not (based on randomization) with the Artemis-generated template, with 10/12-systematic cores throughout the prostate. SBx locations could not encompass the TBx sites, so that the results of each type of biopsy were independent and did not overlap.

Using the BK Ultrasound 5000 MRI-TRUS Fusion platform, fusion target biopsy was performed on the suspicious area previously identified on the multiparametric-MRI using a realtime alignment of the T2-weighted sequence to the TRUS image. MRI-TRUS images alignment was possible due to a tracking device consisted in a sensor coil on the TRUS probe paired with a magnetic field generator to register the location of the tracking device in the 3D space. At least 3 cores were taken for each lesion and the number of additional cores was based on the diameter of the lesion. The number of cores taken was related to the size of the lesions; the cores were carried out along the long axis of the lesion with a maximum of 2 biopsies taken for each needle. TRUS Standard Biopsy was a typical 12 cores double sextant template from lateral to medial of base, mid, and apex. Only the TRUS images, with no multiparametric-MRI target data available, were used for the standard biopsy portion of the case.

\subsection{Pathologic analysis}

Histopathologic examination was carried out by a single dedicated genitourinary pathologist with more than 20 years of experience, who was blinded to the origin (MRI-TRUS TBx or SBx) of each single core. Not indolent Prostate Cancer was defined by the presence of Gleason Score $\geq 7$ (ISUP grade $\geq 2$ ).

\subsection{Study design and endpoints}

After inclusion in each cohort A and B, cases were randomly assigned to an only $\mathrm{TBx}$ strategy versus a $\mathrm{TBx}+\mathrm{SBx}$ strategy. Primary endpoints of this study were overall $\mathrm{PCa}$-detection rate (DR), csPCa-DR, and pathologic results between MRI-TRUS TBx and SBx. Secondary endpoints were correlations between clinical characteristics of the population and csPCa detection on biopsy results.

\subsection{Statistical analysis}

Means, medians, and interquartile ranges were reported for continuous variables. Frequencies and proportions were reported for categorical variables. The Mann-Whitney $U$ test and $\chi^{2}$ tests were used to compare the statistical significance of differences in medians and proportions, respectively. Multivariate logistic regression was performed to evaluate if age, PSA, or PIRADS categorization and type of cohort analyzed (ie, A vs B) were associated with the detection of csPCa at biopsies.
All analyses were carried out using SPSS IBM Statistics v. 22.0 (IBM Corp, Armonk, NY) with level of statistical significance set at $P<.05$.

\section{Results}

A total of 312 patients were included in the 2 cohorts (cohort A: 213 cases; cohort B: 99 cases). All cases were consecutively assigned to MRI-TRUS TBx alone or to a TBx + SBx strategy. The clinical, radiologic, and pathologic characteristics of the entire population are listed in Table 1. No statistically significant differences in terms of age, PSA, PIRADSv2 score distribution, biopsy cores taken per patient by SBx were present between the 2 biopsy strategy groups (Table 1). The 2 biopsy groups were homogeneous regarding most of clinical and radiological data, except for prostate volume (median value 50 vs $40 \mathrm{cc}$; IQR 39.5 61.25 vs $35-50$, respectively) and radiological dimension of the index lesion ( 13 vs $10 \mathrm{~mm}$; IQR $10-16,25$ vs $10-12$, respectively).

Median number of targeted and random cores per patient were respectively 6 (IQR, 4-6) and 11.5 (IQR 10-12). Table 2 shows clinical characteristics of cases on the basis of the pathological diagnosis of csPCa and clinically insignificant $\mathrm{PCa}$. csPCa showed a higher percentage of PI-RADS score $4 / 5$ (73.2\%) when compared with ciPC $(33.3 \%)$.

\subsection{Detection rate of $P C a$ and $c s P C a$}

Table 3 shows the detection rate of all $\mathrm{PCa}, \mathrm{cs} \mathrm{PCa}$, and ratio csPCa/all PCa between patients assigned to MRI-TRUS TBx alone versus $\mathrm{TBx}+\mathrm{SBx}$. Between the 2 groups, no significant differences were found in terms of overall $\mathrm{PCa}$ detection rate (77.6\% vs $69.6 \%$ respectively; $P=.36$ ) and csPCa detection rate ( $48.2 \%$ vs $60.9 \%$ respectively; $P=.12$ ). The MRI-TRUS TB alone cohort showed a higher csPCa/PCa ratio $(87.5 \%$ vs $62.2 \%$; $P=.03$ ) mainly due to the lower number of indolent (ISUP 1) tumor diagnosed.

Moreover, at the MRI-TRUS TB $+\mathrm{SB}$ group subanalysis, a significantly higher csPCa-DR was obtained at the MRI-TRUS TB cores when compared with the SBx cores $(43.7 \%$ vs $24.1 \%$, respectively; $P=.01$ ) (Table 2 ) with a concomitant more accurate Gleason Score stratification (Table 4). Twenty-four out of 81 cases $(29.6 \%)$ were upgraded from benign at $\mathrm{SBx}$ cores to csPCa at TBx cores and 18 out of $51(35.3 \%)$ were upgraded from ciPC at $\mathrm{SBx}$ cores to csPCa at $\mathrm{TBx}$ cores. On the contrary $5 / 65$ cases $(7.7 \%)$ benign and $3 / 33(9.0 \%)$ with ciPC at TBx cores were upgraded to Gleason Score $3+4$ at SBx cores (Table 4).

\subsection{Multivariate analysis}

In adjusted analyses, age, PSA levels, PIRADS score distribution were not significantly associated with csPCa detection at $\mathrm{SBx}$ (Table 5). Therefore, independently of these parameters, either in the rebiopsy (OR $0.43,0.21-0.97$ ) or active surveillance (OR $0.46,0.32-0.89)$ setting, SBx cores were negatively associated with the csPCa-DR when combined to TBx cores.

\section{Discussion}

The advantage of magnetic resonance imaging TBx to SBx in increasing the detection rate of clinical significant prostate cancer, either in naive or in rebiopsy populations, has been well 
Table 1

Clinical, radiologic, and pathological characteristics of the population.

\begin{tabular}{|c|c|c|c|c|c|c|c|}
\hline & All $(n=312)$ & $\begin{array}{c}\text { MRI-TRUS } \\
\text { TBx+ TRUS SBx cohort } \\
(n=174) \\
\end{array}$ & $\begin{array}{c}\text { MRI-TRUS TBX ALONE, } \\
\text { cohort } \\
(n=138)\end{array}$ & $P$ value & $\begin{array}{l}\text { Previous } \\
\text { negative } \\
(n=213)\end{array}$ & $\begin{array}{c}\text { Active } \\
\text { surveillance } \\
(\mathbf{n}=99)\end{array}$ & $P$ value \\
\hline $\begin{array}{l}\text { AGE [y] } \\
\text { Median (IQR) }\end{array}$ & $\begin{array}{l}68 \\
(62-72)\end{array}$ & $\begin{array}{l}67 \\
(61-72)\end{array}$ & $\begin{array}{l}68.5 \\
(62.5-71.5)\end{array}$ & .45 & $\begin{array}{l}68 \\
(62-72)\end{array}$ & $\begin{array}{l}68 \\
(61-71.5)\end{array}$ & .84 \\
\hline $\mathrm{PSA}[\mathrm{ng} / \mathrm{mL}]$ & 7.36 & 7.36 & 7.39 & 0.95 & 7.89 & 6.90 & $*$ \\
\hline Median (IQR) & $(5.21-9.26)$ & $(5.43-9.63)$ & $(4.75-9.22)$ & & $(5.97-10.0)$ & $(4.0-8.22)$ & .02 \\
\hline PSA density $[\mathrm{ng} / \mathrm{mL} / \mathrm{cc}]$ & 0.15 & 0.14 & 0.18 & .15 & 0.15 & 0.15 & .75 \\
\hline Median (IQR) & $(35-57)$ & $(39.5-61.25)$ & $(35-50)$ & .008 & $(40-60)$ & $(32.5-50)$ & .01 \\
\hline Susp. area & 11.5 & 13 & 10 & & 12 & 10 & \\
\hline Diameter [mm] & $(10-15)$ & $(10-16.25)$ & $(10-12)$ & .01 & $(10-17)$ & $(8.5-12)$ & .001 \\
\hline \multicolumn{8}{|l|}{ Median (IQR) } \\
\hline \multicolumn{8}{|l|}{ PI-RADS-v2 n (\%) } \\
\hline $3 / 5$ & $156(50 \%)$ & $93(53.4 \%)$ & $63(45.6 \%)$ & .71 & $105(49.3 \%)$ & $51(51.5 \%)$ & \\
\hline Total & $16(15-18)$ & $16(15-18)$ & - & - & $16(15-18)$ & $16(15-18)$ & .92 \\
\hline Fusion & $6(4-6)$ & $6(4-6)$ & $6(6-7)$ & .91 & $6(4-7)$ & $6(6-6.5)$ & .87 \\
\hline Random & $11.5(10-12)$ & $11.5(10-12)$ & - & - & $11(10-12)$ & $12(10-12)$ & .95 \\
\hline
\end{tabular}

$M R I=$ magnetic resonance imaging, TRUS = trans rectal ultrasound, TBX = targeted biopsy, $S B x=$ systematic biopsy, PSA = prostatic-specific antigen, IQR =interquartile range, $n=$ number.

In bold values that are statistically significant

demonstrated by multicenter studies and stated by international guidelines. ${ }^{[13-15]}$ However, the csPCa yield for TBx alone versus TBx plus SBx after accounting for overlapping of SBx cores with TBx cores, has not been well studied. EAU guidelines in a naive population, when multiparametric magnetic resonance imaging is performed and its PIRADS is $\geq 3$, recommend with a strong level of evidence to combine targeted and systematic biopsies. On the contrary in a prior negative biopsy, when mpMRI is PIRADS $\geq 3$, the recommendation to perform targeted biopsy only, reaches a weak level of evidence. ${ }^{[13]}$ In active surveillance strategy, TBx and SBx appear to be complementary to each other, both missing a significant proportion of cancer upgrading or

Table 2

Characteristics of the entire PCa population in relation to niPC and iPC pathologic diagnosis.

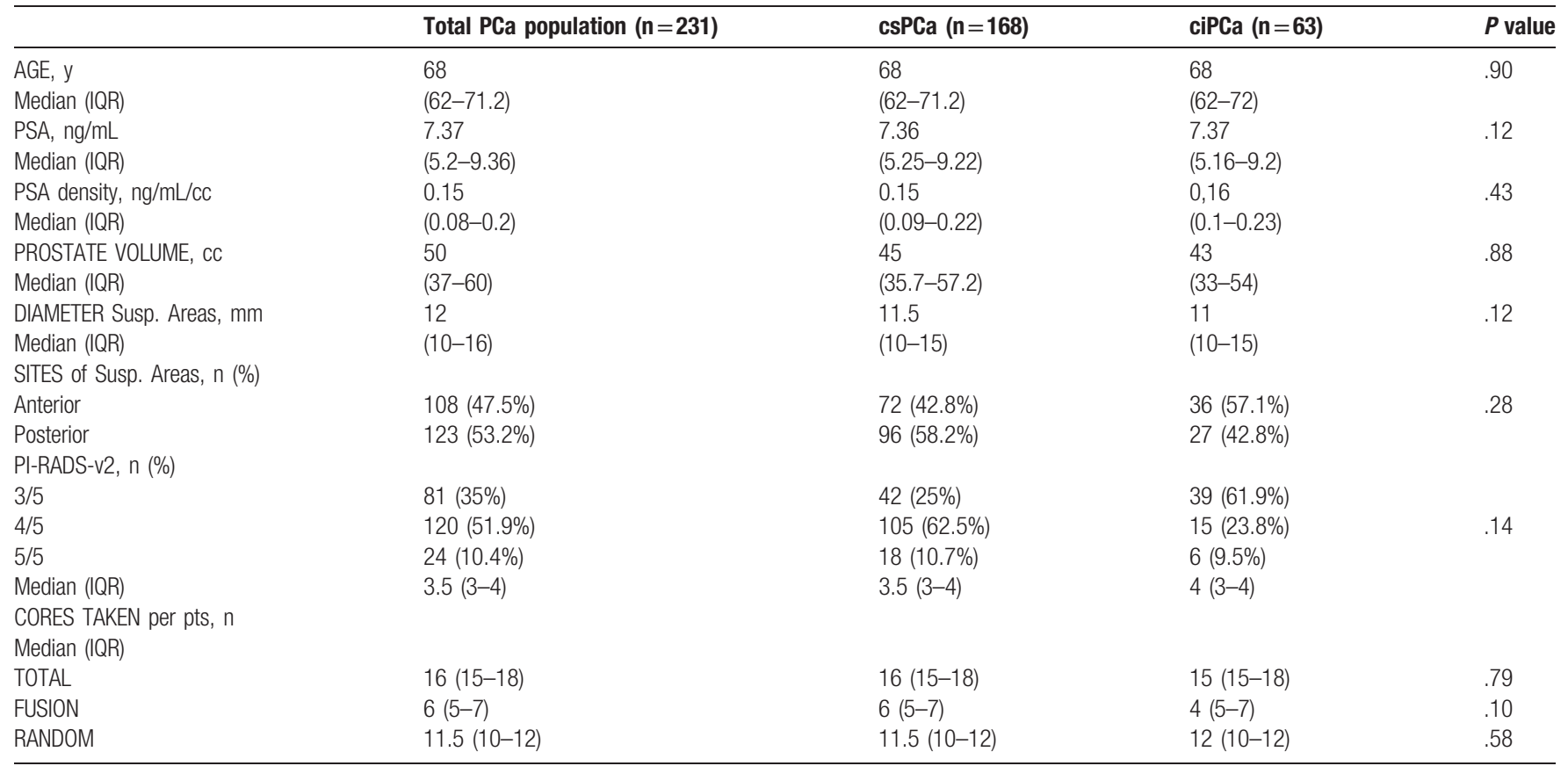

$\mathrm{ciPCa}=$ clinical indolent prostate cancer, $c s \mathrm{PCa}=$ clinically significant prostate cancer, $I Q R=$ interquartile range, $n=$ number, $P S A=$ prostatic-specific antigen. 
Detection rate and histological results.

\begin{tabular}{|c|c|c|c|c|c|c|}
\hline & \multicolumn{3}{|l|}{ Entire population $(n=312)$} & \multicolumn{3}{|c|}{ MRI-TRUS TBX + TRUS SBx cohort $(n=174)$} \\
\hline & $\begin{array}{l}\text { MRI-TRUS TBX + TRUS SBX } \\
(n=174)\end{array}$ & $\begin{array}{l}\text { MRI-TRUS TBX } \\
(n=138)\end{array}$ & $P$ value & MRI-TRUS TBx & TRUS SBX & $P$ value \\
\hline Detection rate $\mathrm{PCa} n(\%)$ & 135/174 (77.6\%) & $96 / 138$ (69.6\%) & .36 & $109 / 174$ (62.6\%) & 93/174 (53.4\%) & .46 \\
\hline Detection rate csPCa $n(\%)$ & $84 / 174(48.2 \%)$ & $84 / 138(60.9 \%)$ & .12 & $76 / 174(43.7 \%)$ & $42 / 174(24.1 \%)$ & .01 \\
\hline $\begin{array}{l}\text { Ratio of detection rate csPCa/PCa n (\%) } \\
\text { ISUP grade } \\
\text { (Gleason score) } n(\%)\end{array}$ & $84 / 135(62.2 \%)$ & $84 / 96(87.5 \%)$ & .03 & $76 / 109$ (69.7\%) & $42 / 93(45.2 \%)$ & .01 \\
\hline $2(3+4)$ & $51(29.3 \%)$ & $21(15.2 \%)$ & & $43(24.7 \%)$ & $33(19.0 \%)$ & \\
\hline $3(4+3)$ & $9(5.2 \%)$ & $24(17.4 \%)$ & & $12(6.9 \%)$ & $0(0 \%)$ & \\
\hline $4(4+4 / 3+5 / 5+3)$ & $12(6.9 \%)$ & $27(19.6 \%)$ & & $12(6.9 \%)$ & $3(1.7 \%)$ & \\
\hline $5(4+5 / 5+4 / 5+5)$ & $12(6.9 \%)$ & $12(8.7 \%)$ & & $9(5.2 \%)$ & $6(3.4 \%)$ & \\
\hline
\end{tabular}

$\operatorname{csPCa}=$ clinically significant prostate cancer, $\mathrm{n}=$ number, $\mathrm{MRI}=$ magnetic resonance imaging, $\mathrm{SBx}=$ systematic biopsy, $\mathrm{TBx}=$ targeted biopsy, TRUS=trans rectal ultrasound. In bold values that are statistically significant

\section{Table 4}

Histological contingency table in the MRI-TRUS TB+TRUS SB cohort.

\begin{tabular}{|c|c|c|c|c|c|c|c|c|}
\hline & \multicolumn{6}{|c|}{ MRI-TRUS TBX } & \multirow{2}{*}{$\begin{array}{l}\text { SBx } \\
\text { Total }\end{array}$} \\
\hline & & Negative & GS $3+3$ & GS $3+4$ & GS 4+3 & GS $4+4$ & GS $4+5$ & \\
\hline \multirow{6}{*}{ SBX } & Negative & 39 & 18 & 6 & 9 & 3 & 6 & 81 \\
\hline & GS $3+3$ & 21 & 12 & 15 & 0 & 3 & 0 & 51 \\
\hline & GS $3+4$ & 5 & 3 & 22 & 0 & 3 & 0 & 33 \\
\hline & GS 4+3 & 0 & 0 & 0 & 0 & 0 & 0 & 0 \\
\hline & GS 4+4 & 0 & 0 & 0 & 0 & 3 & 0 & 3 \\
\hline & GS 4+5 & 0 & 0 & 0 & 3 & 0 & 3 & 6 \\
\hline \multicolumn{2}{|c|}{$\begin{array}{c}\text { MRI-TRUS TBx } \\
\text { Total }\end{array}$} & 65 & 33 & 43 & 12 & 12 & 9 & 174 \\
\hline
\end{tabular}

$\mathrm{GS}=$ Gleason score, $\mathrm{MRI}=$ magnetic resonance imaging, $\mathrm{SBx}=$ systematic biopsy, TBx=targeted biopsy, TRUS =trans rectal ultrasound.

\section{Table 5}

Multivariate logistic regression predicting the presence of niPC on SBx cores.

\begin{tabular}{lll}
\hline Variable & OR (95\%) & $\boldsymbol{P}$ value \\
\hline Age & $1.03(0.99-1.15)$ & .068 \\
PSA & $1.06(1.01-1.13)$ & .124 \\
PIRADSv2 score & $0.99(0.96-1.01)$ & .059 \\
Re-biopsy cohort & $0.43(0.21-0.97)$ & .041 \\
AS cohort & $0.46(0.32-0.89)$ & .039 \\
\hline
\end{tabular}

$A S=$ active surveillance, $\mathrm{OR}=$ odds ratio, $\mathrm{PSA}=$ prostatic-specific antigen, $\mathrm{PI}-\mathrm{RADS}=$ prostate imaging-reporting and data system, v2 = version 2 .

reclassification. Thus, combining the 2 biopsy techniques seems to be the best way to select patients for AS or to monitoring them. ${ }^{[19,20]}$ However, EAU guidelines recommend to perform mpMRI before confirmatory biopsy with a strong level of evidence, but the recommendation to perform the combination of TBx and SBx at confirmatory biopsy reaches a weak level of evidence.

For these reasons we decided to consider for our study 2 different populations (prior negative biopsy and active surveillance) in which the level of evidence to combine SBx and TBx is weak. In addition, we excluded the naive biopsy population, in which this level of evidence is strong.

The purpose of our study was to investigate the potential benefit in terms of Detection Rate and pathological stratification of prostate cancer using a contextual SBx during an MRI-TRUS TBx.

In our experience, independently of other clinical parameters, either in the rebiopsy or in the active surveillance setting, SBx cores were negatively associated with the csPCa detection rate when combined to TBx cores. In fact, in both populations, the MRI-TRUS TBx alone cohort showed a higher csPCa/PC ratio $(87.5 \%$ vs $62.2 \% ; P=.03)$ mainly due to the lower number of indolent (ISUP 1) tumor diagnosed.

Considering the group of patients submitted to a combination of MRI-TRUS targeted and systematic cores, SBx upgraded TBx only in $7.7 \%$ with benign and $9.0 \%$ with ciPC at TBx cores and the upgrade was to a Gleason score $3+4$ (ISUP 2).

Main limitation of our study is not equally distributed population among the 2 cohort enrolled; therefore, we were not able to establish a clear difference in the outcomes reached.

Our analysis was prospective, and the 2 cohorts are representative of the normal clinical practice. Our findings suggest that MRI-TRUS TBx represents the elective method to perform prostate biopsy in these 2 settings and the combination 
of a SBx does not improve the detection rate of csPCa nether in a population of prior negative biopsy nor in AS confirmatory biopsy.

\section{Author contributions}

Conceptualization: Angelo Porreca, Matteo Ferro, Ottavio de Cobelli, Ettore De Berardinis, Gian Maria Busetto.

Data curation: Daniele Romagnoli, Paolo Corsi, Alessandro Del Rosso.

Formal analysis: Francesco Del Giudice, Gian Maria Busetto. Investigation: Daniele D’Agostino, Daniele Romagnoli.

Methodology: Paolo Corsi, Alessandro Del Rosso, Martina Maggi, Alessandro Sciarra.

Project administration: Angelo Porreca, Riccardo Schiavina.

Resources: Daniele D’Agostino, Martina Maggi, Alessandro Sciarra.

Software: Francesco Del Giudice, Marco Giampaoli.

Supervision: Angelo Porreca, Giuseppe Lucarelli, Riccardo Schiavina.

Validation: Benjamin I. Chung, Matteo Ferro, Ettore De Berardinis.

Visualization: Matteo Ferro, Riccardo Schiavina, Alessandro Sciarra.

Writing - original draft: Francesco Del Giudice, Marco Giampaoli, Matteo Ferro, Gian Maria Busetto.

Writing - review \& editing: Martina Maggi, Benjamin I. Chung, Ottavio de Cobelli, Giuseppe Lucarelli, Riccardo Schiavina, Ettore De Berardinis, Alessandro Sciarra, Gian Maria Busetto.

\section{References}

[1] Siegel R, Naishadham D, Jemal A. Cancer statistics 2012. CA Cancer J Clin 2012;62:10-21.

[2] Vagnoni V, Bianchi L, Borghesi M, et al. Adverse features and competing risk mortality in patients with high-risk prostate cancer. Clin Genitourin Cancer 2017;15:e239-48.

[3] Dickinson L, Ahmed HU, Allen C, et al. Magnetic resonance imaging for the detection, localisation, and characterisation of prostate. Eur Urol 2011;59:477-94.

[4] Panebianco V, Sciarra A, De Berardinis E, et al. PCA3 urinary test versus $1 \mathrm{H}-\mathrm{MRSI}$ and DCEMR in the detection of prostate cancer foci in patients with biochemical alterations. Anticancer Res 2011;31:1399-405.

[5] Vagnoni V, Brunocilla E, Bianchi L, et al. State of the art of PET/CT with 11choline and 18F-fluorocholine in the diagnosis and follow-up of localized and locally advanced prostate cancer. Arch Esp Urol 2015;68:354-70.

[6] Watanabe Y, Terai A, Araki T, et al. Detection and localization of prostate cancer with the targeted biopsy strategy based on ADC map: a prospective large-scale cohort study. J Magn Reson Imaging 2012;35:1414-21.

[7] Numao N, Yoshida S, Komai Y, et al. Usefulness of pre-biopsy multiparametric magnetic resonance imaging and clinical variables to reduce initial prostate biopsy in men with suspected clinically localized prostate cancer. J Urol 2013;190:502-8.

[8] Siddiqui MM, Rais-Bahrami S, Truong H, et al. Magnetic resonance imaging/ultrasound fusion biopsy significantly upgrades prostate cancer versus systematic 12-core transrectal ultrasound biopsy. Eur Urol 2013;64:713-9.

[9] Hoeks CM, Schouten MG, Bomers JG, et al. Three-Tesla magnetic resonance-guided prostate biopsy in men with increased prostate-specific antigen and repeated, negative, random, systematic, transrectal ultrasound biopsies: detection of clinically significant prostate cancers. Eur Urol 2012;62:902-9.

[10] Wegelin O, van Melick HHE, Hooft L, et al. Comparing three different techniques for magnetic resonance imaging-targeted prostate biopsies: a systematic review of in-bore versus magnetic resonance imagingtransrectal ultrasound fusion versus cognitive registration is there a preferred technique? Eur Urol 2017;71:517-31.

[11] van Hove A, Savoie PH, Maurin C, et al. Comparison of image-guided targeted biopsies versus systematic randomized biopsies in the detection of prostate cancer: a systematic literature review of well-designed studies. World J Urol 2014;32:847-58.

[12] Schoots IG, Roobol MJ, Nieboer D, et al. Magnetic resonance imagingtargeted biopsy may enhance the diagnostic accuracy of significant prostate cancer detection compared to standard transrectal ultrasound guided biopsy: a systematic review and meta-analysis. Eur Urol 2015;68:438-50.

[13] Mottet N, Bellmunt J, Bolla M, et al. EAU-ESTRO-SIOG Guidelines on Prostate Cancer. Part 1: Screening, Diagnosis, and Local Treatment with Curative Intent. Eur Urol 2017;71:618-629.

[14] Kasivisvanathan V, Rannikko AS, Borghi M, et al. MRI-targeted or standard biopsy for prostate-cancer diagnosis. N Engl J Med 2018; 378:1767-77.

[15] Rouvière O, Puech P, Renard-Penna R, et al. Use of prostate systematic and targeted biopsy on the basis of multiparametric MRI in biopsy-naive patients (MRI-FIRST): a prospective, multicentre, paired diagnostic study. Lancet Oncol 2019;20:100-9.

[16] Grasso AA, Cozzi G, De Lorenzis E, et al. Multicenter analysis of pathological outcomes of patients eligible for active surveillance according to PRIAS criteria. Minerva Urol Nefrol 2016;68:237-41.

[17] Rosenkrantz AB, Verma S, Choyke P, et al. Prostate magnetic resonance imaging and magnetic resonance imaging targeted biopsy in patients with a prior negative biopsy: a consensus statement by AUA and SAR. J Urol 2016;196:1613-8.

[18] Weinreb JC, Barentsz JO, Choyke PL, et al. PI-RADS prostate imaging_reporting and data system: 2015, version 2. Eur Urol 2016; 69:16-40.

[19] Schoots IG, Nieboer D, Giganti F, et al. Is magnetic resonance imagingtargeted biopsy a useful addition to systematic confirmatory biopsy in men on active surveillance for low-risk prostate cancer? A systematic review and meta-analysis. BJU Int 2018;122:946-58.

[20] Klotz L, Loblaw A, Sugar L, et al. Active surveillance magnetic resonance imaging study (ASIST): results of a randomized multicenter prospective trial. Eur Urol 2019;75:300-9. 